SECTION 31. Economic research, finance, innovation.

Naumov Anatoly Aleksandrovich

Docent, Candidate of Technical Sciences, Center of Applied Mathematical Research, Novosibirsk, Russia, E-mail: A_A_Naumov@mail.ru

\title{
RISK ANALYSIS AND INVERSE PROBLEMS FOR INVESTMENT PROJECTS INVESTIGATION
}

In this paper approaches to risk areas desired values and parameters of investment projects estimating are proposed and investigated.

Keywords: Investment projects, efficiency, risks, inverse problems.

\section{УДК 330.46:330.322.5: 658.155}

\section{АНАЛИЗ РИСКОВ И ОБРАТНЫЕ ЗАДАЧИ ДЛЯ ИССЛЕДОВАНИЯ ИНВЕСТИЦИОННЫХ ПРОЕКТОВ}

В работе предложены и исследованы подходы к оцениванию областей желаемых значений рисков и параметров инвестиционных проектов (ИП).

Ключевые слова: инвестиционные проекты, эффективность, риски, обратные задачи.

В основу предлагаемых методов положены идеи обратных преобразований пространств значений показателей эффективности ИП и их рисков, которые реализуются с использованием алгоритмов обхода границы и дискретизации областей (см. [1]).

Модели бизнес-процессов - удобное представление ИП для решения задач, связанных с оцениванием и оптимизацией их рисков. Риски ИП, представимых бизнеспроцессами $\overparen{B P}_{s}(t)$ (см. [1]) обусловлены неопределенностями в знании значений характеристик бизнес-процессов и их параметров $\pi$ (значений банковских ставок, цен и т.д.). Условимся бизнес-процессы с неопределенностями в потоках и в других характеристиках обозначить через $\widetilde{B P}=\left\{\widetilde{B P}_{i}(t)\right\}, i=1,2, \ldots, N$, и, соответственно, для их параметров - через $\tilde{\pi}=\left\{\tilde{\pi}_{i}\right\}, i=1,2, \ldots, N$. Очевидно, такая неопределенность в знании характеристик ИП (и соответствующих им бизнес-процессов) приводит к тому, что вместо вектора показателей (номинального, планового) $\vec{Q}$ (или $\left\{\vec{Q}_{(i)}\right\}, i=1,2, \ldots, M$ ) реально будем иметь дело с вектором $\overrightarrow{\widetilde{Q}}$ (или с векторами $\left\{\overrightarrow{\widetilde{Q}}_{(i)}\right\}, i=1,2, \ldots, M$ ). Величина отклонения вектора $\vec{Q}$ от вектора $\overrightarrow{\widetilde{Q}}$ и будет характеризовать риск ИП. Схема, характеризующая переход от пары $\langle\widetilde{B P}, \tilde{\pi}\rangle$ к вектору рисков $\vec{R}(t)$, может быть изображена таким образом:

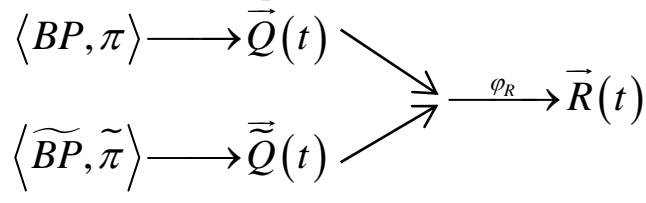


Индивидуальными рисками можно воспользоваться, если объединить их в пары с самими показателями, например, вида $\left\langle Q_{i, n o m}, R_{j, i}\right\rangle, i \in\{1,2, \ldots, M\}, j=1,2, \ldots, K, j$ номер (индекс) вида риска или $\left\langle Q_{i, n o m} ;\left\{R_{j, i}\right\}, j=1,2, \ldots, K\right\rangle, i \in\{1,2, \ldots, M\}$, если каждому из показателей сопоставить множество оценок рисков $\left\{R_{j, i}\right\}, j=1,2, \ldots, K$, ему соответствующих. Такие пары порождают характеристики бизнес-процессов вида $\vec{Q}_{R}=\left(Q_{1 R}, Q_{2 R}, \ldots, Q_{M R}\right)^{T}$, где $Q_{i R}=\left\langle Q_{i, \hat{i} \hat{i} i} ;\left\{R_{j, i}\right\}, j=1,2, \ldots, K\right\rangle, i \in\{1,2, \ldots, M\}$.

Оценивание (нахождение) областей $R_{Q}$ и $R_{\pi}$ (областей рисков) в пространствах показателей $\overrightarrow{\widetilde{Q}}$ и параметров $\pi$ позволяет учесть их при управлении ИП и повысить эффективность такого управления.

Важной для практики представляется задача оценивания желаемых и допустимых областей в пространстве параметров $\pi$ по известным областям в пространстве показателей $\vec{Q}$. Как и для задачи оценивания области $R_{\pi}$ по области $R_{Q}$, когда требуется найти обратное преобразование для $\overparen{B P}_{s}^{\pi, \vec{Q}}$ (обозначим его условно через $\left(\overparen{B P}_{s}^{\pi, \vec{Q}}\right)^{-1}$ ) и, таким образом, найти прообраз в пространстве параметров $\pi$ области $R_{Q}$, т.е. $R_{\pi}=\left(\overparen{B P}_{s}^{\pi, \vec{Q}}\right)^{-1}\left(R_{Q}\right)$, так и в данном случае области желаемых значений параметров $\pi($ $D_{\pi}$, от английского «Desirable») и допустимых значений этих параметров $\left(P_{\pi}\right.$, от английского «Permissible»). Элементы этих множеств будем обозначать через $\pi_{i}^{0} \in D_{\pi}, i=1,2, \ldots, p_{d}, \quad$ и $\quad$ через $\pi_{i}^{\diamond} \in P_{\pi}, i=1,2, \ldots, p_{p}, \quad$ соответственно. Для соответствующих областей в пространстве показателей $\overrightarrow{\widetilde{Q}}$ введем обозначение $D_{Q}$ (для множества желаемых значений $\overrightarrow{\widetilde{Q}}$ ) и $P_{Q}$ (для множества допустимых значений $\overrightarrow{\widetilde{Q}}$ ). Соответственно элементы этих двух множеств будем обозначать через $\overrightarrow{\widetilde{Q}}_{i}^{o} \in D_{Q}, i=1,2, \ldots, q_{d}$, и $\overrightarrow{\widetilde{Q}}_{i}^{\triangleleft} \in P_{Q}, i=1,2, \ldots, q_{p}$. Тогда задача по поиску областей $D_{\pi}$ и $P_{\pi}$ сводится к нахождению прообразов областей $D_{Q}$ и $P_{Q}$ относительно отображения $\overparen{B P}_{s}^{\pi, \bar{Q}}$ . На Рис. проиллюстрированы множества $D_{Q}, P_{Q}, D_{\pi}, P_{\pi}$ и отображение $\overparen{B P}_{s}^{\pi, \widehat{Q}}$. 


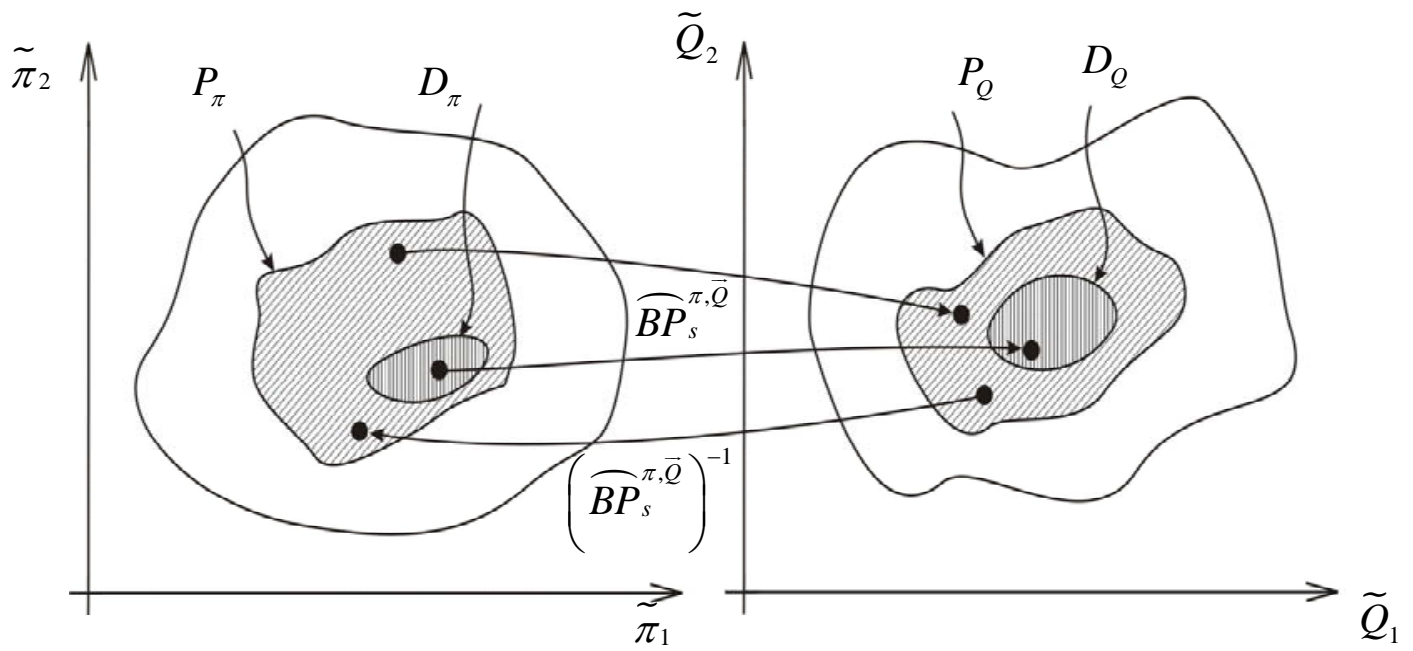

Рисунок 1 - Множества $D_{Q}, P_{Q}, D_{\pi}, P_{\pi}$ и отображение $\overparen{B P}_{s}^{\pi, \vec{Q}}$.

Формально эти множества связаны между собой соотношениями $\overbrace{B P}^{\pi, \vec{Q}}\left(D_{\pi}\right)=D_{Q}$ и $\overparen{B P}_{s}^{\pi, \vec{Q}}\left(P_{\pi}\right)=P_{Q}$. Поскольку на практике известны (задаются, назначаются) области $P_{Q}$ и $D_{Q}$ в пространстве показателей, то, для того чтобы найти соответствующие им области в пространстве параметров, требуется найти обратное отображение для $\overparen{B P}_{s}^{\pi, \vec{Q}}$ и с его помощью оценить области $P_{\pi}$ и $D_{\pi}:\left(\overparen{B P}_{s}^{\pi, \vec{Q}}\right)^{-1}: P_{Q} \rightarrow P_{\pi}$ и $\left(\overparen{B P}_{s}^{\pi, \vec{Q}}\right)^{-1}: D_{Q} \rightarrow D_{\pi}$. Задача нахождения областей $P_{\pi}$ и $D_{\pi}$ по известным областям $P_{Q}$ и $D_{Q}$ относится к классу так называемых обратных задач. На основе таких преобразований могут быть решены задачи по нахождению: областей для рисков ИП; областей упущенной выгоды; областей сильной и слабой чувствительности ИП; областей сильной и слабой маневренности ИП и т.д.

\section{Литература}

1. Наумов А.А. Теоретические и прикладные вопросы моделирования бизнеспроцессов. Модели, алгоритмы, программы: Монография/ А. А. Наумов. - LAP LAMBERT Academic Publishing, 2012. - 464 c.

[https://www.ljubljuknigi.ru/store/ru/book/Tеоретические-и-прикладные-вопросымоделирования-бизнес-процессов/isbn/978-3-8383-6534-3]

2. Список трудов [Электронный ресурс]. URL: https://sites.google.com/site/ anatolynaumov2011/home/spisok-trudov-list-of-papers (дата обращения: 25.11.2013). 